\title{
INTERACTIVE OPERATIONAL PLANNING PROCEDURE OF THE TECHNICAL OPERATIONS IN THE COMPOUND MULTIOPERATIONAL MANUFACTURING
}

\author{
S.Ya. Fomin, Dr. Eng., Professor of the Chair of ACS \\ Yu.V. Silakova, Student
}

National University of Science and Technology "MISIS” (MISIS) (Moscow, Russia)

E-MAIL: stan.fomin2010@yandex.ru

Abstract. The structure of interactive operational planning procedure of the technological cold-shaped pipes manufacturing is presented. Generation and search of acceptable schedules of loading of workplaces are realized by using the genetic algorithm, allowing quickly enough to solve complex semistructured optimization challenges in a mode close to a mode of real time.
Keywords: interactive procedure, genetic algorithm, technological route (track), mathematical model.

\section{REFERENCES}

1. Frolov E.B., Zagidullin R.R. MES-sistemy. Vid «sverhu», vzgljad iznutri. 12 news: Novosti korporativnyh informacionnyh sistem i prilozhenij (View "from above", a look inside. 12 news: News of corporate information systems and applications). Available at URL: http://erpnews.ru/doc2689.html (Accessed 20.01.2013)

2. F o m in S.Ja. Izvestija VUZov. Chernaja metallurgija. 2013. № 3. Pp. $48-55$.

3. Fom in S.Ja., B eljakova Ju.S. Izvestija VUZov. Chernaja metallurgija. 2013. № 5. Pp. $58-62$.

Received November 12, 2013

УДК 621.365.2

\section{МАТЕМАТИЧЕСКОЕ МОДЕЛИРОВАНИЕ ФОРМЫ ДУГ ПРИ ИХ ЭЛЕКТРОМАГНИТНОМ ВЗАИМОДЕЙСТВИИ. СООБЩЕНИЕ 2}

\author{
И.М. Ячиков, д.т.н. профессор кафедры «Вычислительная техника и программирование» \\ E.M. Костылева, инженер-программист управления информационных технологий и АСУ
}

Магнитогорский государственный технологический университет (Магнитогорск, Россия)

\begin{abstract}
Аннотация. Предложена математическая модель и алгоритм расчета для нахождения коэффициентов полинома, описывающего формы осей электрических дуг для случаев двух или трех дуг постоянного тока или среднего положения дуг трехфазного переменного тока, горящих между электродами и токоподводящей поверхностью. Создано программное обеспечение, позволяющее приближенно рассчитать формы осей столбов при электромагнитном взаимодействии двух и трех дуг. Приведены результаты моделирования для аргоновых дуг. Установлено, что при взаимодействии дуг с протекающими по ним одинаковыми токами, форма оси столба дуги зависит от рода тока, количества дуг и расстояния между ними и слабо зависит от значения силы тока.
\end{abstract}

Ключевые слова: электрическая дуга, трехфазный переменный ток, токопроводящая поверхность, форма электрической дуги, электромагнитное взаимодействие.

E-MAIL: jachikov@mail.ru

В настоящее время в металлургической промышленности используется множество различных печей, в которых источником тепла служат электрические дуги постоянного или трехфазного переменного тока промышленной частоты. Эти печи могут использовать несколько одновременно горящих дуг и отличаться количеством электродов и родом используемого тока. Так, в дуговых и плазменных печах источниками тепла могут являться две или три дуги постоянного тока. В дуговых сталеплавильных печах, рудотермических и рудовосстановительных печах, питаемых трехфазным током, одновременно могут гореть три и даже шесть дуг.

При конструировании дуговых и плазменных печей необходимо учитывать форму дуг, зависящую как от условий их горения и теплообмена, так от электромагнитных сил, которые определяются величиной проте- кающих токов, длиной дуг, расстоянием между ними и конструкцией токоподводов. В работах $[1,2]$ рассмотрен случай электромагнитного взаимодействия двух дуг постоянного тока, горящих между параллельными катодами и токоподводящей поверхностью. Получены дифференциальные уравнения и предложены алгоритмы для их решения и определения формы электрических дуг [3 - 8]. Однако электромагнитное взаимодействие двух дуг постоянного тока не охватывает всех возможных вариантов взаимного влияния нескольких дуг, встречающихся в промышленных агрегатах.

Целью работы является разработка алгоритмов расчета и моделирование формы осей двух или трех электрических дуг постоянного или трехфазного переменного токов, горящих между электродами и токоподводящей поверхностью. 
Электромагнитное взаимодействие дуг определяется уравнением равновесия элемента столба дуги $[1,2]$

$$
d \vec{F}_{1-2}+d \vec{F}_{1-1}+d \vec{F}_{1-\amalg}=0,
$$

где $d \vec{F}_{1-2}$ - сила взаимодействия элемента дуги с другой дугой (или другими дугами), $d \vec{F}_{1-1}-$ сила взаимодействия элемента дуги с самим собой, $d \vec{F}_{1-ц}-$ центробежная сила, стремящаяся выпрямить столб дуги.

Рассмотрим электромагнитное взаимодействие двух дуг, через которые протекают постоянные или переменные токи.

Характер взаимодействия двух дуг, через которые протекают постоянные токи $I_{1}, I_{2}$, представлен на рис. 1 . Вектор электромагнитной силы $d \vec{F}_{1-2}$, действующей на элемент дуги $d \vec{l}_{1}$ со стороны второй дуги, находится в плоскости $y \mathrm{Oz}$. Он перпендикулярен $d \vec{l}_{1}$, для случая однонаправленных токов имеет направление, показанное на рис. $1, a$, для случая противоположно направленных токов направление, показанное на рис. 1,6 , а его модуль равен [1]

$$
\left|d \vec{F}_{1-2}\right|=\chi I_{1} I_{2} d \vec{l}_{1}
$$

где $\chi=\frac{\mu_{0}}{4 \pi} \int_{0}^{l}\left(\frac{\frac{d y(\xi)}{d \xi}(\xi-z)-(y(\xi)-y(z))}{r_{21}^{3}}\right) d \xi$.

При однонаправленных постоянных токах $\left(I_{1} I_{2}>0\right)$ дуги притягиваются, в противном случае $\left(I_{1} I_{2}<0\right)$ отталкиваются. Если токи не меняются, то при отклонении дуги от положения равновесия возникают силы, стремящиеся ее вернуть в исходное состояние, поэтому электрическая дуга имеет определенную фиксированную форму, напоминающую геометрическую дугу.

Электромагнитное взаимодействие двух дуг, через которые протекают гармонические токи со сдвигом фаз $2 \pi / 3: i_{1}=i_{01} \sin \frac{2 \pi}{T} \tau$ и $i_{2}=i_{02} \sin \left(\frac{2 \pi}{T} \tau \pm \frac{2 \pi}{3}\right)$, где $T-$ период колебаний тока; $i_{01}, i_{02}$ - амплитудные значения токов. При рассмотренном сдвиге фаз $2 \pi / 3$ дуги испытывают сложный колебательный процесс, но средняя действующая сила приводит к их отталкиванию друг от друга.

В этом случае на каждый элемент дуги действует электромагнитная сила, вектор которой находится в плоскости $y O z$, он перпендикулярен $d \vec{l}_{1}$ и имеет направление, показанное на рис. 1, б. Характерной особенностью электромагнитного взаимодействия электрических дуг переменного тока является непрерывность изменения формы и размеров дуги за счет воздействия знакопеременной периодической силы с периодом $T / 2$ (рис. 2), ее мгновенное значение $d \vec{F}_{1-2}=\chi i_{1} i_{2} d \vec{l}_{1}$.

Дуги, как легкоподвижные элементы, совершают колебательное движение с частотой $2 / T$ вокруг положения, определяемого среднеинтегральным значением силы за период

$$
d \tilde{F}_{1-2}=\chi d l_{1} \frac{1}{T} \int_{0}^{T} i_{1} i_{2} d \tau=-\frac{i_{01} i_{02}}{4} \chi d l_{1}=-0,5 i_{\text {д1 }} i_{\text {д2 }} \chi d l_{1},
$$

где $i_{\text {д1 }}, i_{\text {д2 }}$ - действующие значения токов.

Сравнивая выражения (2) и (3) видно, что две дуги, через которые протекают переменные токи с действующими значениями $i_{\text {д1 }}, i_{\text {д2 }}$, испытывают такое же среднее электромагнитное взаимодействие, как две дуги, через которые протекают постоянные токи $I_{1}=i_{\text {д1 }}, I_{2}=i_{\text {д2 }}$ противоположного направления с коэффициентом пропорциональности $k_{1}=0,5$.

Рассмотрим электромагнитное взаимодействие трех дуг, через которые протекают постоянные или переменные токи, причем вертикальные электроды, к которым привязаны дуги, образуют в плане равносторонний треугольник со стороной $L=0,5 D \sqrt{3}$, где $D$ - диаметр распада электродов.

Рассмотрим электромагнитное взаимодействие трех дуг постоянного тока, причем токи имеют одинаковое направление и величину $\left(I_{1} I_{2}>0, I_{2} I_{3}>0\right.$, $\left.I_{1}=I_{2}=I_{3}=I\right)$. В этом случае на элемент дуги действуют две силы $d \vec{F}_{1-2}$ и $d \vec{F}_{1-3}$, направление которых показано на рис. $3, a$. Результирующая сила $d \vec{F}_{1-2-3}$

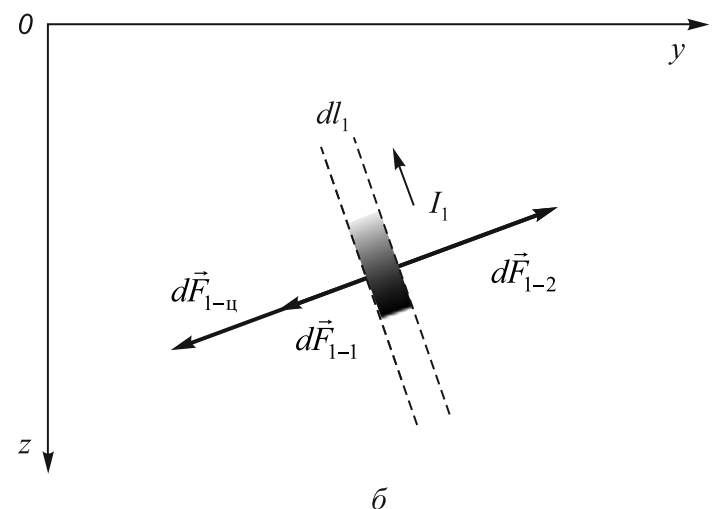

Рис. 1. Электромагнитное взаимодействие элементов двух дуг, через которые протекают:

$a$ - однонаправленные постоянные токи; $\sigma$ - противоположно направленные постоянные или переменные токи со сдвигом фаз $2 \pi / 3$ 


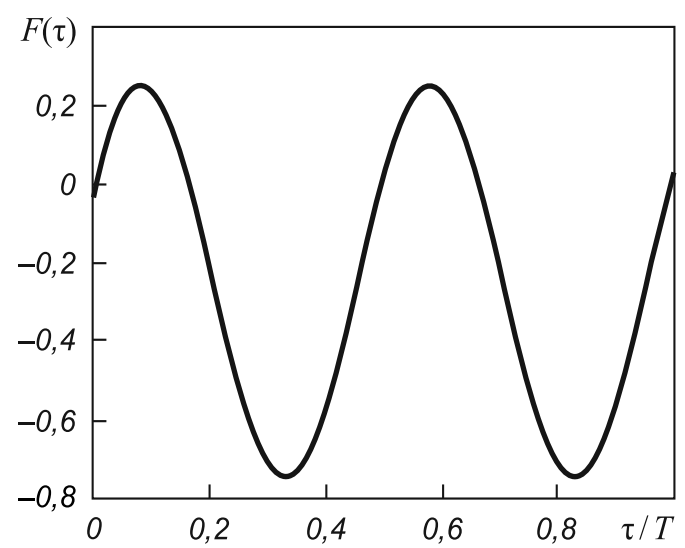

Рис. 2. Мгновенное значение электромагнитной силы парного $a$ взаимодействия дуг за период

перпендикулярна элементу $d \vec{l}_{1}$ и направлена к центру распада электродов, а ее модуль равен $\left|d \vec{F}_{1-2-3}\right|=$ $=\sqrt{d F_{1-2}^{2}+d F_{1-3}^{2}+2 d F_{1-2} d F_{1-3} \cos (\pi / 3)}$. Модули значений силы $d F_{1-2}$ и $d F_{1-3}$ равны и определяются уравнением (2), поэтому

$$
d F_{1-2-3}=\sqrt{3} d F_{1-2}=\sqrt{3} \chi I^{2} d l_{1} .
$$

Три дуги, через которые протекают постоянные токи, проявляют такое же электромагнитное взаимодействие, как между двумя дугами, через которые протекают постоянные сонаправленные токи с коэффициентом пропорциональности $k_{1}=\sqrt{3}$.

Рассмотрим случай электромагнитного взаимодействия трех дуг с одинаковыми постоянными токами, причем ток первой дуги направлен в противоположную сторону двум другим токам. На элемент первой дуги действуют две отталкивающиеся силы $d \vec{F}_{1-2}$ и $d \vec{F}_{1-3}$ (рис. $3, \sigma$ ). Результирующая сила $d \vec{F}_{1-2-3}$ перпендикулярна элементу $d \vec{l}_{1}$ и направлена от центра распада электродов, а ее модуль равен $d F_{1-2-3}=\sqrt{3} \chi I^{2} d l_{1}$.

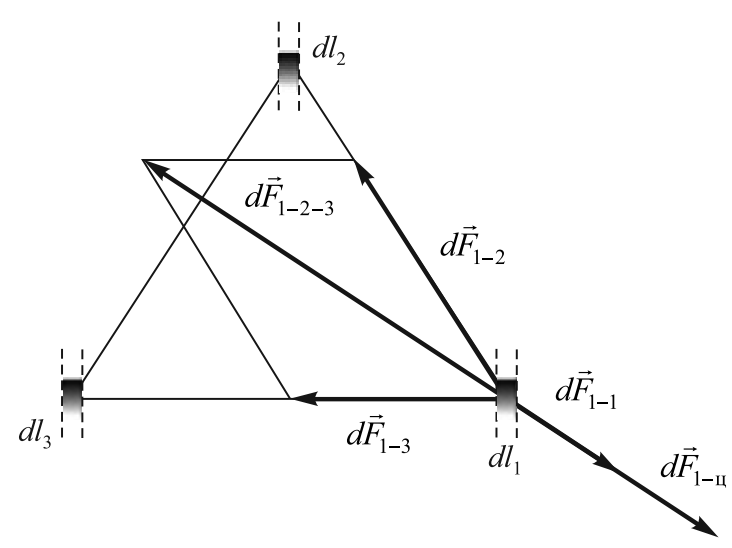

$a$
На элемент второй и третьей дуги действует сила, перпендикулярная элементу $d \vec{l}_{1}$ и направленная по касательной к окружности с центром распада электродов, а ее модуль равен $d F_{2-1-3}=\sqrt{3} \chi I^{2} d l_{1}$.

Электромагнитное взаимодействие на дугу постоянного тока от двух других дуг, через которые протекают постоянные токи в противоположную сторону, такое же, как между двумя дугами, через которые протекают постоянные противоположно направленные токи с коэффициентом пропорциональности $k_{1}=\sqrt{3}$.

Электромагнитное взаимодействие трех дуг, через которые протекают гармонические токи со сдвигом фаз $2 \pi / 3: i_{1}=i_{01} \sin \frac{2 \pi}{T} \tau, i_{2}=i_{02} \sin \left(\frac{2 \pi}{T} \tau+\frac{2 \pi}{3}\right)$ и $i_{3}=i_{03} \sin \left(\frac{2 \pi}{T} \tau-\frac{2 \pi}{3}\right)$, где $i_{01}, i_{02}, i_{03}$ - амплитудные значения токов [9]. Рассмотрим случай, когда действующее значение токов одинаковое $i_{\text {д1 }}=i_{\text {д2 }}=i_{\text {д3 }}=i_{\text {д }}$.

При электромагнитном взаимодействии трех переменных токов на элемент $d \vec{l}_{1}$ действуют две силы отталкивания $d F_{1-2}=\chi i_{1} i_{2} d l_{1}$ и $d F_{1-3}=\chi i_{1} i_{3} d l_{1}$, направление которых показано на рис. $3, \sigma$. Направление результирующей силы $d \vec{F}_{1-2-3}$ меняется с течением времени и совпадает с вектором $d \vec{F}_{1-2}+d \vec{F}_{1-3}$. Годограф силы воздействия на элемент дуги показан на рис. 4. Модуль результирующей силы равен

$$
d F_{1-2-3}=\sqrt{3} d F_{1-2}=\sqrt{3} F_{0}\left|\sin \frac{2 \pi}{T} \tau\right| d l_{1},
$$

где $F_{0}=\frac{\chi i_{\text {д }}^{2}}{2}$.

Поскольку мгновенные значения силы $d F_{1-2-3}$ непрерывно меняются в течение каждого полупериода, то изменяются по величине и направлению действующие на элемент дуги электродинамические усилия. Найдем среднеинтегральную силу за половину периода, определяющую среднее положение дуги:

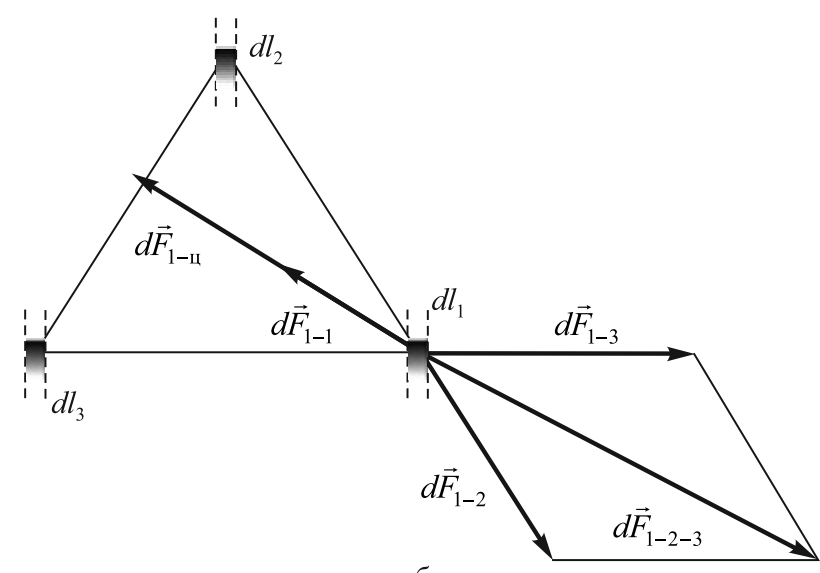

6

Рис. 3. Схема электромагнитного взаимодействия элемента тока $d \vec{l}_{1}$ с элементами токов двух других дуг, расположенных в вершинах равностороннего треугольника, через все элементы протекают:

$a$ - однонаправленные постоянные токи; $\sigma$ - постоянные токи, при этом ток через элемент $d \vec{l}_{1}$ направлен в противоположную сторону двум другим, либо переменные токи со сдвигом фаз $2 \pi / 3$ 


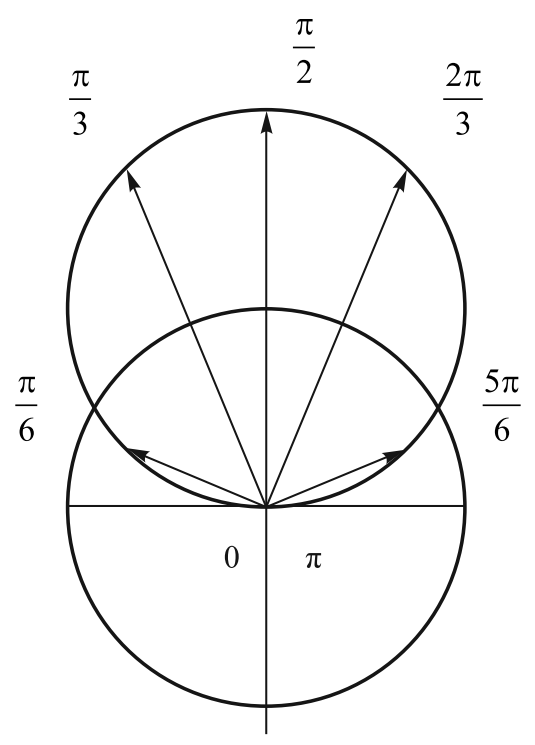

Рис. 4. Изменение направления и величины усилий, воздействующих на элемент дуги, от двух других переменных токов в течение одного полупериода

$$
\begin{aligned}
d \tilde{F}_{1-2-3} & =-\frac{2 i_{\mu}^{2}}{T} \frac{\chi \sqrt{3}^{3}}{2} \int_{0}^{\frac{T}{2}} \sin \left(\frac{2 \pi}{T} \tau\right) d \tau d l_{1}= \\
& =-\frac{\sqrt{3}}{\pi} i_{\text {д }}^{2} d l_{1} \approx-0,551 i_{\text {д }}^{2} d l_{1} .
\end{aligned}
$$

Эта сила направлена от центра распада электродов.

Три дуги, через которые протекают переменные токи с действующим значением тока $i_{\text {д }}$, проявляют такое же электромагнитное взаимодействие как две дуги, через которые протекают постоянные противоположно направленные токи с коэффициентом пропорциональности $k_{1}=\sqrt{3} / \pi$.

Рассмотрим электромагнитное взаимодействие на элемент дуги $d \vec{l}_{1}$ постоянного тока со стороны тока самой дуги. Вектор $d \vec{F}_{1-1}$ находится в плоскости $y O z$, перпендикулярен $d \vec{l}_{1}$ и противоположно направлен вектору $d \vec{F}_{1-2}$ (см. рис. $\left.1, \sigma\right)$, а его модуль равен [1]

$$
\begin{aligned}
\left|d \vec{F}_{1-1}\right|=\frac{\mu_{0} I_{1}^{2}}{4 \pi} \int_{0}^{l} & \frac{\left(\frac{d y(\zeta)}{d \zeta}(\zeta-z)-(y(\zeta)-y(z))\right)}{r_{31}^{3}} \times \\
& \times d \zeta d l_{1}=\chi_{1} I_{1}^{2} d l_{1} .
\end{aligned}
$$

При электромагнитном взаимодействии гармонического тока $i_{1}=i_{01} \sin \left(\frac{2 \pi}{T} \tau+\varphi\right)$ с самим собой среднеинтегральное значение силы

$$
\begin{gathered}
d F_{1-1}=\frac{\chi_{1}}{T} \int_{0}^{T} i_{1}^{2} d \tau=\frac{\chi_{1} i_{01}^{2}}{T} \int_{0}^{T}\left(\sin \frac{2 \pi}{T} \tau+\varphi\right)^{2} d \tau d l_{1}= \\
=\frac{\chi_{1} i_{01}^{2}}{2} d l_{1}=\chi_{1} i_{\text {ม1 }}^{2} d l_{1} .
\end{gathered}
$$

Видно, что электромагнитное взаимодействие постоянного тока с самим собой такое же, как переменного с таким же действующим значением.

В общем случае уравнение (1) равновесия элемента столба дуги при его электромагнитном взаимодействии с одной или двумя дугами постоянного или переменного токов можно записать как

$$
k_{1} d \vec{F}_{1-2}+d \vec{F}_{1-1}+d \vec{F}_{1-\text { L }}=0 .
$$

Значения коэффициента $k_{1}$ и характер электромагнитного взаимодействия при различных случаях сведены в таблице.

На основе алгоритмов, предложенных в работах $[1,2,7$-9], для решения уравнения (8) создана компьютерная программа «Взаимодействие дуг», позволяющая моделировать форму осей столбов двух или трех взаимодействующих электрических дуг постоянного или трехфазного переменного токов, горящих между электродами и токоподводящей поверхностью (Ячиков И.М., Костылева Е.М. Свидетельство о государственной регистрации программы № 2013619388 от 03.10.2013). В качестве исходных данных задаются теплофизические свойства плазмообразующего газа, геометрические размеры и параметры токов дуг. В процессе работы программы выдаются значения промежуточных невязок и результирующие коэффициенты полиномов, описывающих форму дуг.

При моделировании взаимодействия дуг с одинаковыми токами было установлено, что их форма практически не зависит от силы тока, но существенно зависит от количества электродов, расстояния между ними и рода тока. Например, снижение расстояния между электродами в 2 раза (с 40 до 20 см) приводит к уменьшению минимального расстояния между дугами практически в 3 раза. Это качественно соответствует результатам, полученным в работе [2].

Проведено моделирование формы двух и трех аргоновых дуг при их электромагнитном взаимодействии при одинаковом значении постоянных и действующих токов 9 кА. При расчетах для аппроксимации формы дуги использовались полиномы третьей степени. На рис. 5. показана форма оси столбов дуги при взаимодействии двух и трех дуг постоянного и переменного токов. Из него видно, что дуги, по которым протекают сонаправленные постоянные или переменные токи, притягиваются или отталкиваются соответственно, причем, чем меньше расстояние между электродами, тем сильнее, и их форма слабо зависит от количества электродов. Дуги, по которым протекают противоположно направленные постоянные токи отталкиваются, и чем меньше расстояние между электродами, тем более значительно, причем форма дуг определяется и количеством электродов.

Bbl6odbl. Предложена математическая модель и алгоритм расчета для нахождения коэффициентов поли- 

Коэффициент $k_{1}$ в уравнении (8) при электромагнитном взаимодействии дуг
постоянного или переменного токов

\begin{tabular}{|c|c|c|}
\hline Токи & $k_{1}$ & $\begin{array}{c}\text { Характер электромагнитного } \\
\text { взаимодействия дуг }\end{array}$ \\
\hline \multicolumn{3}{|c|}{ Взаимодействие двух дуг } \\
\hline Постоянные токи, текущие в одном направлении & 1 & Притягиваются \\
\hline Постоянные токи, текущие в разных направлениях & 1 & Отталкиваются \\
\hline Переменные токи со сдвигом фаз $\varphi=0$ & 1 & Притягиваются \\
\hline Переменные токи со сдвигом фаз $\varphi=2 \pi / 3$ & 0,5 & Отталкиваются \\
\hline \multicolumn{3}{|c|}{ Взаимодействие трех дуг (электроды образуют равносторонний треугольник) } \\
\hline Постоянные токи, текущие в одном направлении & $\sqrt{3}$ & $\begin{array}{c}\text { Притягиваются к центру распада } \\
\text { электродов }\end{array}$ \\
\hline $\begin{array}{c}\text { Постоянные токи, ток первый первой дуги имеет } \\
\text { противоположное направление второму и третьему } \\
\text { току }\end{array}$ & $\sqrt{3}$ & $\begin{array}{c}\text { Первый ток отталкивается от центра } \\
\text { распада электродов; второй и третий } \\
\text { направлены по касательной к окружности } \\
\text { с центром распада электродов }\end{array}$ \\
\hline Переменный трехфазный ток & $\frac{\sqrt{3}}{\pi}$ & $\begin{array}{c}\text { Отталкиваются от центра распада } \\
\text { электродов }\end{array}$ \\
\hline
\end{tabular}
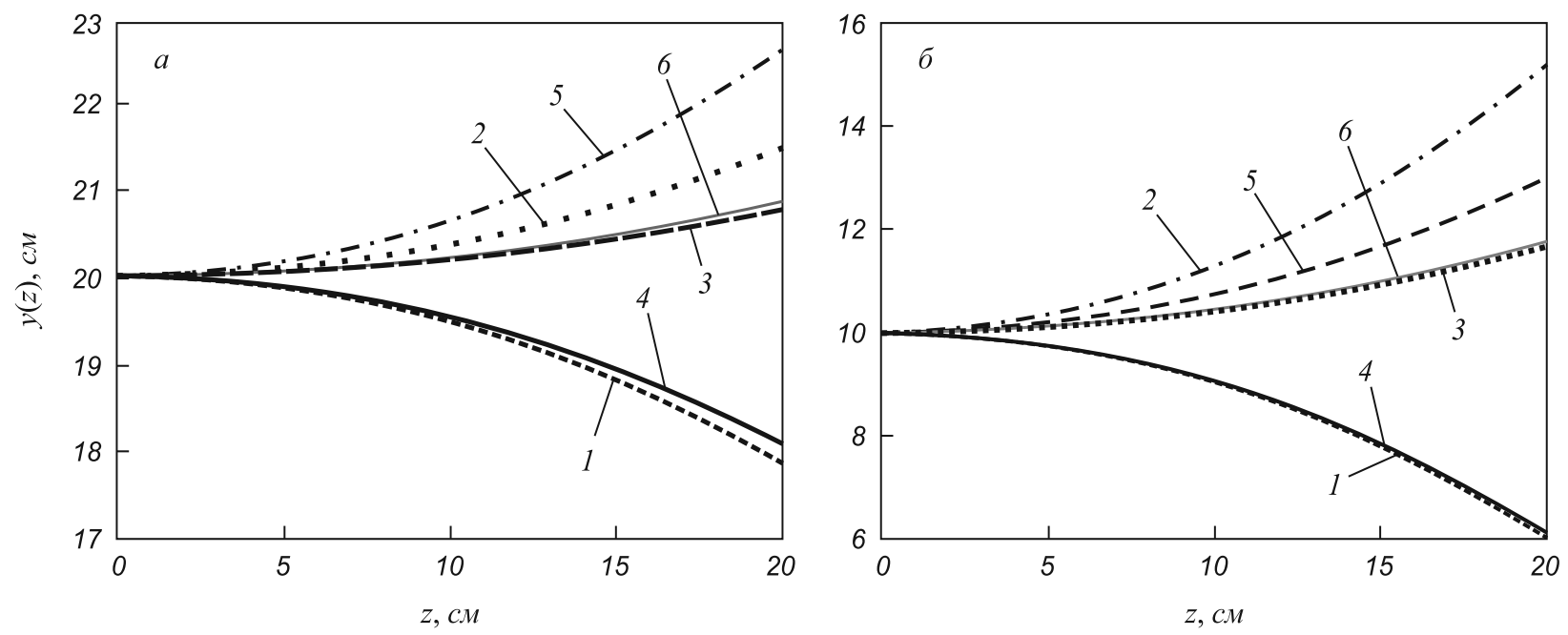

Рис. 5. Форма оси столба дуги при взаимодействии двух дуг, по которым протекают:

1 - постоянные токи, текущие в одном направлении; 2 - постоянные токи, текущие в разных направлениях;

3 - переменные токи, сдвиг фаз между которыми $2 \pi / 3$.

Форма оси столба дуги при взаимодействии трех дуг, по которым протекают:

4 - постоянные токи, текущие в одном направлении; 5 - постоянные токи, один из которых имеет противоположное направление; 6 - переменные токи, сдвиг фаз между которыми $2 \pi / 3$.

Значение постоянных и действующих токов 9 кА; расстояние между электродами:

$$
a-40 \mathrm{~cm} ; \sigma-20 \mathrm{~cm}
$$

нома, описывающего формы осей электрических дуг для случаев двух или трех дуг постоянного тока или среднего положения дуг трехфазного переменного тока, горящих между электродами и токоподводящей поверхностью. Создано программное обеспечение, позволяющее приближенно рассчитать формы осей столбов при электромагнитном взаимодействии двух и трех дуг.

Установлено, что при взаимодействии дуг с протекающими по ним одинаковыми токами, форма оси столба дуги зависит от рода тока, количества дуг и расстояния между ними и слабо зависит от значения силы тока. Созданное программное обеспечение может использоваться для анализа тепловой работы элементов конструкции металлургических агрегатов, где источником нагрева являются несколько свободно горящих дуг. Кроме этого, с ее помощью можно провести оценки неравномерности теплового износа футеровки и графитированных электродов.

\section{БИБЛИОГРАФИЧЕСКИЙ СПИСОК}

1. Я ч и ко в И.М., Ко с тыл е в а Е.М. // Изв. вуз. Черная металлургия. 2014. № 1. С. $59-64$. 
2. Бортничук Н.И, Крутянский М.М. Плазменно-дуговые плавильные печи. - М.: Энергоиздат, 1981. - 120 с.

3. Я ч и ко в И.М., Ко с тыл е в а Е.M. Моделирование формы дуг постоянного тока при их электромагнитном взаимодействии: Межрегион. сб. науч. тр. /Под ред. В.М. Колокольцева. Вып. 11. - Магнитогорск: МГТУ, 2011. С. 195 - 201.

4. Азбелев Н.В., Макси мов В.П., Рахматуллина Л.Ф. Элементы современной теории функционально-дифференциальных уравнений. Методы и приложения. - М.: Институт компьютерных исследований, 2002. - 304 с.

5. Д митриев С.С., Кузнецов Е.Б. // Журнал вычислительной математики и математической физики. 2008. № 3. C. $430-444$.
6. В е р жби цк и й В.M. Численные методы. Математический анализ и обыкновенные дифференциальные уравнения. - М.: Высшая школа, 2001. - 383 с.

7. Ячиков И.М., Костылев а Е.М. Информационные технологии и системы: Матер. Первой Междунар. конф. - Челябинск: Изд-во ЧелГУ, 2012. С. $36-38$.

8. Я чи ко в И.М., Ко с тыл ев а Е.М. Теория и практика тепловых процессов в металлургии: Сб. док. Междунар. научнопрактич. конф. - Екатеринбург: УрФУ, 2012. С. 423 - 426.

9. Я чи ков И.М., З ар ецкая Е.М. // Изв. вуз. Черная металлургия. 2011. № 1. С. $18-20$.

(C) 2014 г. И.М. Ячиков, Е.М. Костылева Поступила 5 марта 2014 г.

\section{THE MATHEMATICAL MODELING OF ARCS' FORM DURING THEIR ELECTROMAGNETIC COUPLING. MESSAGE 2}

\section{I.M. Yachikov, Dr. Eng., Professor of the Chair of computer science and programming \\ E.M. Kostyleva, Software Engineer of department of infor- mation technology management and control systems}

Magnitogorsk State Technical University (Magnitogorsk, Russia)

E-MAIL: jachikov@mail.ru

Abstract. The mathematical model and software algorithms for finding of polynomial describing forms of electrical arcs axes in cases of two or three arcs of direct current or middle situation of arcs of three-phase alternating current burning among electrodes and current-carrying surface were proposed. The software which allows approximately calculate forms of axes of arcs' columns in case of electromagnetic coupling of two or three arcs. Results of the modeling for argon arcs are represented. It has been established that in the case of arcs' coupling with equal currents the form of axis of arc column depends on the kind of the current, number of arcs and distance among them and slightly depends on amperage.

Keywords: electric arc, three-phase alternating current (three-phase AC), current-carrying surface, form of the electric arc, electromagnetic coupling.

\section{REFERENCES}

1. J a chikov I.M., K o s ty lev a E.M. Izvestija VUZov. Chernaja metallurgija, 2014. № 1. Pp. 59 - 64.

2. Bortnichuk N.I, Krutjanskij M.M. Plazmenno-dugovye plavil'nye pechi (Plasma arc furnaces). Moscow: Jenergoizdat, 1981. $120 \mathrm{p}$.

3. Jachikov I.M., Kostyleva E.M. Modelirovanie formy dug postojannogo toka pri ih jelektromagnitnom vzaimodejstvii: mezhregion. sb. nauch. tr. (Shape modeling arcs DC at their elec- tromagnetic interaction: interregional collection of sci. works). Kolokol'cev V.M. ed. Issue 11. Magnitogorsk: Izd-vo Magnitogorsk. gos. tehn. un-ta. Pp. $195-201$.

4. Azbelev N.V., Maksimov V.P., Rahmatullina L.F. Jelementy sovremennoj teorii funkcional'no - differencial'nyh uravnenij. Metody i prilozhenija (Elements of the modern theory of functional - differential equations. Methods and Applications). Moscow: Institut komp'juternyh issledovanij, 2002. 304 p.

5. Dmitriev S.S., Kuznecov E.B. Zhurnal vychislitel'noj matematiki i matematicheskoj fiziki. 2008. № 3. Pp. 430 - 444.

6. Verzhbickij V.M. Chislennye metody. Matematicheskij analiz $i$ obyknovennye differencial'nye uravnenija (Numerical methods. Mathematical analysis and ordinary differential equations). Moscow: Vyssh. shk., 2001. 383 p.

7. Jachikov I.M., Kosty leva E.M. Polozhenie jelektricheskih dug pri ih jelektromagnitnom vzaimodejstvii v mnogojelektrodnyh dugovyh pechah. Informacionnye tehnologii i sistemy: materialy Pervoj mezhdunar. konf. (Position of electric arcs when the electromagnetic interaction in multi-electrode arc furnaces, in Information Technologies and Systems: Proceedings of the First Intern. Conf.). Cheljabinsk: Izd-vo Cheljab. gos. un-ta, 2012. Pp. $36-38$.

8. Jachikov I.M., Kostyleva E.M. Priblizhennyj algoritm nahozhdenija polozhenija jelektricheskih dug pri ih jelektromagnitnom vzaimodejstvii v mnogojelektrodnyh dugovyh pechah. Teorija $i$ praktika teplovyh processov $v$ metallurgii: sbornik dokladov mezhdunarodnoj nauchno-prakticheskoj konferencii (An approximate algorithm for finding the position of electric arcs when the electromagnetic interaction in multi-electrode arc furnaces, in Theory and Practice of thermal processes in metallurgy: a collection of reports of International Scientific-practical Conf.). Ekaterinburg: UrFU, 2012. Pp. $423-426$.

9. J a ch i kov I.M., Z a r e k a j a E.M. Izvestija VUZov. Chernaja metallurgija. 2011. № 1. Pp. 18 - 20.

Received March, 5, 2014 